\title{
The role of arousal in boundary judgement errors
}

\author{
Deanne M. Green ${ }^{1}$ - Jessica A. Wilcock ${ }^{1} \cdot$ Melanie K. T. Takarangi ${ }^{1}$
}

Published online: 19 March 2019

(C) The Psychonomic Society, Inc. 2019

\begin{abstract}
Eyewitnesses to a crime rely heavily on their visual memory; however, there are many ways that the details of visual scenes can be missed, or distorted. In particular, for emotional scenes, the "boundaries" are narrowed at retrieval, whereas central detailssuch as a weapon - are remembered in greater detail. This phenomenon is known as boundary restriction, the reverse of boundary extension whereby people tend to expand the boundaries of a neutral scene at retrieval. In the present series of experiments, we investigated whether arousal is the element of an emotional scene that leads to increased boundary restriction or reduced boundary extension. We presented neutral images to participants either with or without a stress-inducing noise. In Experiment $1 \mathrm{a}$ and $1 \mathrm{~b}$, at test, participants viewed the image they originally viewed next to the same image but with narrower or wider boundaries and selected which of the two images they originally viewed. In Experiment 2, at test, participants viewed the identical image they originally viewed, but were told the boundaries had been changed. Participants selected the extent to which the images at test had restricted or extended boundaries compared to their memory of the original image. When the noise stressor was present, participants made more boundary restriction errors - selecting the image with narrower boundaries than the original - and fewer boundary extension errors than when the noise was absent. Our data suggest that arousal plays a key role in boundary judgements.
\end{abstract}

Keywords Recognition $\cdot$ Arousal $\cdot$ Memory $\cdot$ Attention $\cdot$ Scene perception

Eyewitnesses to a crime rely on visual memory - underpinned by where their attention was directed during the crime-when they recount what they saw. However, details of visual scenes can be missed, or distorted, affecting an eyewitness's memory report. In particular, for emotional scenes, we know that central details - such as a weapon - are often remembered better than peripheral details - such as other witnesses (Kramer, Buckhout, \& Eugenio, 1990). This effect has been described as memory narrowing, tunnel vision, or boundary restriction, contingent on the specific materials and paradigm used (Berntsen, 2002; Candel, Merckelbach, \& Zandbergen, 2003; Fawcett, Russell, Peace, \& Christie, 2013; Mathews \& Mackintosh, 2004; Safer, Christianson, Autry, \& Osterlund, 1998). Here, our focus is on boundary restriction, whereby people erroneously remember the boundaries of a scene as narrower than they actually were. One explanation for boundary restriction highlights enhanced

Melanie K. T. Takarangi

melanie.takarangi@ flinders.edu.au

1 School of Psychology, Flinders University, GPO Box 2100, Adelaide, South Australia 5001, Australia attention or memory processing resulting from increased arousal (Mathews \& Mackintosh, 2004; Safer et al., 1998). Previous research has compared scenes rated as differing in valence and, thus, presumably arousal. Of course, there are other qualities that could make an image inherently more or less memorable, such as content, context, or composition. Moreover, some studies fail to demonstrate boundary restriction, even using similar paradigms (e.g., Candel et al., 2003). Thus, in the present study we aimed to isolate arousal from the other qualities of a scene, to examine its influence on boundary restriction.

Memory for visual information is highly malleable. Boundary extension, the tendency to remember more expansive background of scenes, is one example of memory malleability. In an early study, Intraub, Gottesman, Willey, and Zuk (1996) showed participants an image of a teddy bear on a single step. When participants drew the scene from memory, they generally drew the teddy bear sitting on a flight of stairs. Boundary extension occurs across age and gender (see Seamon et al., 2002); despite prior warning (Intraub \& Bodamer, 1993); with both real-life images (Intraub \& Bodamer, 1993; Intraub et al., 1996) and abstract shapes (McDunn, Siddiqui, \& Brown, 2014); and when memory is assessed by drawing, forced-choice, or boundary adjustment 
tests (where participants adjust an image's boundaries; Chapman, Ropar, Mitchell, \& Ackroyd, 2005; Daniels \& Intraub, 2006; Dickinson \& Intraub, 2009; Intraub, 2004; Intraub, Hoffman, Wetherhold, \& Stoehs, 2006).

It was initially hypothesized that boundary extension occurs because we extrapolate beyond the periphery of a scene to contextualize scene information into the wider world (e.g., Gottesman \& Intraub, 1999). However, more recent work suggests boundary extension is a source-monitoring error (Intraub, 2010, 2012). Specifically, people rely on internally generated mental representations instead of their sensory experience. Visual-sensory input is only available for a short duration, and because of constraints to the visual system - for example, vision is pieced together from a series of quick eye movementsimagined details are then extrapolated from incomplete sensory inputs (Blackmore, Brelstaff, Nelson, \& Troscianko, 1995; Simons \& Levin, 1998). Memory for this extrapolated information is difficult to distinguish from sensory information derived from the actual stimulus, resulting in a source-monitoring error (Intraub, 2012). In Munger and Multhaup (2016), participants with higher (vs. lower) spatial abilities made more boundary extension errors (i.e., they judged images they had seen earlier as appearing more wide angled-remembering the images as having wider boundaries - even though the images were unchanged). These data suggest boundary errors could occur because of source-monitoring errors; specifically because of internally generated mental representations that involve visuospatial elaboration.

Attention allocation also appears to affect boundary judgements. When attention is divided by a visual search task - and hence diverted away from the scene(s) - participants tend to rate test images as having narrower borders (a form of boundary extension error: participants remember the original images as having wider borders compared with test) compared with participants without an attentional distractor (Intraub, Daniels, Horowitz, \& Wolfe, 2008). Furthermore, people tend to make less expansive boundary extension errors for the left compared with the right side of images - when maintaining central fixation on briefly presented images (Dickinson \& Intraub, 2009). This finding is consistent with pseudoneglect, whereby neurotypical people pay less attention to the right (vs. left) side of their visual field (Bowers \& Heilman, 1980). Together, these findings suggest decreased attention to scene content can increase boundary extension; by contrast, increased attention can attenuate boundary extension.

Interestingly, our visual system prioritizes attention to highly arousing or stressful stimuli, at the expense of neutral, nonstressful stimuli (Lang, 2010; Mather, 2007). For example, we know that stimuli signalling threat- such as a weaponcan make people more attentive to the source of that threat (Gilbert, 2001; see also Fawcett et al., 2013; Kramer et al., 1990). When negative stimuli draw attention away from other nonnegative information, this process may reduce or eliminate broader contextualization of the scene, making it less likely that an internally generated mental representation will be confused with actual sensory information (Vuilleumier, 2005). Indeed, some research shows boundary extension is attenuated when the scenes are negative, such as when they depict threatening objects, physical violence, and death (Levine \& Edelstein, 2009; Mathews \& Mackintosh, 2004; Safer et al., 1998). Put differently, after viewing emotionally negative or traumatic images, people sometimes show boundary restriction, erroneously recall boundaries as narrower than they were - or indeed make fewer boundary extension errors (Christianson \& Loftus, 1987; Mathews \& Mackintosh, 2004; Ménétrier, Didierjean, \& Vieillard, 2013; Safer et al., 1998; Takarangi, Oulton, Green, \& Strange, 2015; Waring, Payne, Schacter, \& Kensinger, 2010).

In one example, participants viewed scenes of actors expressing particular emotions (Ménétrier et al., 2013). Participants then saw the same scenes again, but were told the camera distance had changed for some images. They indicated on a 5-point scale whether the image was more zoomed-in, more zoomed-out, or the same as the original image. Participants displayed more boundary extension errorsjudging scenes as closer at test-for positively valenced emotions (happiness, pleasure), than for the negatively valenced emotions (anger, irritation). Thus, negative stimuli may attenuate boundary extension. However, in the absence of a neutral condition it is also possible that the positive images enhanced boundary extension. In another example, participants viewed either negative or neutral single images, or a series of slides depicting a story ending with a negative or neutral scene (Safer et al., 1998). Safer et al. (1998) used two different methods to test participants' memory for the images: a forced-choice paradigm, with the original image accompanied by three distracters (all more zoomed-in than the original, or two zoomed-in and one more zoomed-out than the original) and a camera-distance judgement. On the forced-choice test, participants selected which image they originally viewed. Participants who viewed negative images made more boundary restriction errors-indicating the image was more zoomed-in than the one they originally saw - than boundary extension errors-indicating the image was more zoomed-out than the one they originally saw. Participants who viewed neutral images made more boundary extension errors than boundary restriction errors. Participants made fewer boundary extension errors for negative images than neutral images. However, they were equally likely to make boundary restriction errors for negative and neutral images.

Yet not all studies have found boundary restriction - or attenuated boundary extension - for negatively valenced stimuli across different test types. Similar to Safer et al. (1998), using the camera-distance paradigm, Candel et al. (2003) found no difference in the rate of boundary extension, and no evidence for increased boundary restriction for negative 
versus neutral images. In a second experiment, Candel et al. asked participants to draw the images from memory. Overwhelmingly, participants made boundary extension errors regardless of valence - drawing the images with the central object smaller and with more extensive boundaries than the original images. Using a camera-distance paradigm, Beighley, Sacco, Bauer, Hayes, and Intraub (2018) also found that valence —operationalized here as positive (happiness, joy, pleasure, social connection) or negative (depression, grief, angst, social isolation) emotion expressed by an actor in each image - did not affect the rate of boundary extension errors.

Together, these studies show that evidence for boundary restriction - and attenuation of boundary extension - is inconsistent. One possibility is that arousal, rather than negative valence per se, affects boundary restriction, and previous results are mixed because relying on incidental arousal (e.g., to an existing stimulus, which could vary in content, context, or composition) rather than using a manipulation of arousal, means that arousal levels vary across studies. Indeed, there is converging evidence that attention narrows specifically when people are highly aroused. An early example demonstrated that a simulated state of arousal - where the effect of stress hormones was mimicked by methamphetamine administration - reduced participants' ability to encode peripheral information beyond their direct line of sight (Callaway \& Dembo, 1958). Further, in a series of studies, participants viewed slide shows that ended in one of two ways: with either a neutral (e.g., woman riding a bicycle) or arousing critical slide (e.g., woman with a head injury; Christianson \& Loftus, 1991; Christianson, Loftus, Hoffman, \& Loftus, 1991). Participants who viewed the arousing slide recalled more central details than those who viewed the neutral slide. Additionally, Echterhoff and Wolf (2012) manipulated arousal at encoding using a video (a stressful burglary or a nonstressful burglary). Participants in the stressful condition exhibited both a greater memory for central details, and a reduced memory for peripheral details. Together, these results suggest that arousal during encoding induces a focus of attention.

To date, there is only indirect evidence that arousal per se affects boundary errors. Mathews and Mackintosh (2004) found that high-anxious participants made more boundary restriction errors than low-anxious participants for highly negative pictures. People with high trait anxiety are more prone to higher states of arousal than those with low trait anxiety (Mathews \& MacLeod, 1994). Thus, if participants with higher trait anxiety were more aroused at encoding, these data might suggest that arousal affects boundary judgments (albeit not independent of valence in this instance). Unfortunately, because the researchers did not report state arousal, we cannot confirm this supposition. Thus, no study has rigorously manipulated arousal to induce boundary judgment errors. Our studies address this important gap.
We manipulated arousal using an external stressor (noise blasts). This paradigm elicits a similar level of psychological and physiological arousal to a fear response (Rhudy \& Meagher, 2001). Across three experiments, we used noise blasts to influence participants' encoding of neutral images. We used neutral images to rule out valence effects. Participants viewed cropped or uncropped versions of images either with or without a noise. In Experiments $1 \mathrm{a}$ and 1b, participants completed a forced-choice recognition test where they saw the image they originally viewed alongside a comparison version. If the participant saw a cropped image at encoding, the test comprised the original cropped image and a version that was zoomed-out with greater boundaries. Incorrectly selecting the zoomed-out version was a boundary extension error. Where participants saw an uncropped image at encoding, the test comprised the original uncropped image and a version that was zoomed-in with reduced boundaries. Incorrectly selecting the zoomed-in version was a boundary restriction error. In Experiment 2, we changed this test to a camera-distance paradigm. We predicted that boundary restriction errors were more likely to occur for images paired with noise than images without noise. We also predicted that boundary extension errors would be less likely to occur for images presented with noise.

\section{Pilot study}

\section{Stimuli preparation}

We wanted to establish distinct semantic categories of images so that our arousal manipulation would be isolated to the category presented with the noise. Dunsmoor, Murty, Davachi, and Phelps (2015) demonstrated that participants could be conditioned to associate external arousal with a particular category of images: participants anticipated arousal for images that were semantically related (e.g., from the same image category of "tools") to images that were earlier presented with an arousing stimulus (a shock). We identified 203 images from the International Affective Picture System (IAPS; Lang, Bradley, \& Cuthbert, 2008) and the Nencki Affective Picture System (NAPS; Marchewka, Zurawski, Jednorog, \& Grabowska, 2014) with a normed emotional valence rating ( $4-6$ on a scale where $1=$ very unpleasant, $9=$ very pleasant $)$ across five possible categories - nature, everyday objects, people, tools, and scenes. Fifty participants from Amazon Mechanical Turk rated how well each image fit into each category-for example, "How well does this image fit into the category: nature?" $(1=$ a very good example, $7=$ a very poor example; adapted from Rosch, 1973). We excluded images rated as highly associated with more than one category (e.g., nature and everyday objects). We found that the nature and everyday-objects categories had the most images with 
unique category membership. We excluded images that did not contain a central object on a wider background, or that we could not crop without cropping the central object in the image. We chose 12 images per category with the best category membership rating and cropped each image to $75 \%$ of its original size and then resized it, which resulted in two versions of each image (i.e., $75 \%$ cropped and $100 \%$ uncropped).

\section{Experiment 1a}

\section{Method}

Participants No previous boundary error research has directly manipulated arousal. Thus, using $\mathrm{G}^{*}$ Power, we calculated the sample size required to detect a small-medium within-subjects effect ( $d=0.30$ ) of arousal on boundary judgements (planned comparisons of noise vs. no noise on boundary extension errors, and on boundary restriction errors). At alpha $=.05$, power $=.80$, the recommended sample size is 90 . This sample size is consistent with previous experiments investigating boundary restriction, which have used 80-150 participants (Mathews \& Mackintosh, 2004; Safer et al., 1998; Takarangi et al., 2015; though we note Anderson, Kelley, \& Maxwell, 2017). To enable our counterbalance - which required divisibility by four - we increased the target sample size to 96 . We recruited 99 participants with normal or corrected-to-normal colour vision from an undergraduate research participation pool, but excluded three participants due to technical errors. Most $(78 \%)$ were female, and identified as Caucasian (including White; 66.7\%). Participants ranged in age from 18 to 46 years $(M=23.08, S D=6.07)$. Participants received course credit, or payment (AUD\$10) for their time.

Design We used a 2 (noise: noise, no noise) $\times 2$ (encoded image: cropped, uncropped) $\times 2$ (category-noise combination: noise-nature, noise-object) mixed design where noise and encoded image were within-subjects and category-noise combination was between-subjects. Our key dependent variable was the proportion of images on which participants made errors.

\section{Materials}

Anxiety measures We measured participants' trait and state anxiety using the using the 20-item trait and state subscales of the State-Trait Anxiety Inventory (STAI-T, STAI-S; Spielberger, Gorsuch, \& Lushene, 1970). Trait anxiety refers to those prone to anxiety, while state anxiety refers to feelings of unpleasantness, such as stress, fear, and discomfort. For STAI-T, participants read a series of statements about how they feel generally (e.g., I am "calm, cool and collected"), and rated how often they felt this way on a 4-point Likert scale. For STAI-S, participants read a series of statements about how they felt at the present time, (e.g., "I feel calm") and rated how much they agreed with each statement on a 4point Likert scale. We measured state anxiety over time, because state anxiety is malleable (unlike trait anxiety). Overall, the STAI has good reliability and validity for both trait and state subscales ( $\alpha=0.88$; Grös, Antony, Simms, \& McCabe, 2007). We also found high internal consistency for STAI-S: $\alpha$ $=0.91$ at Time 1 , and 0.93 at Time 2 .

Manipulation of noise White noise above 90 decibels (dB), in short bursts, briefly increases stress, fear, and surprise similar to that of emotional arousal (Rhudy \& Meagher, 2001). We administered a 95-98 $\mathrm{dB}$ range of white noise with headphones for the full 2 seconds that each image was presented. The noise was paired with one category of images (i.e., nature/ everyday objects), which was counterbalanced so that both categories of images were presented with noise in the sample.

\section{Procedure}

The Flinders University's Social and Behavioural Research Ethics Committees approved this research. Participants completed the experiment on an Apple iMac desktop computer using SuperLab. Participants were situated approximately $40 \mathrm{~cm}$ from the screen (see Fig. 1).

Encoding phase After informed consent procedures, participants completed demographic questions, the STAI-T ${ }^{1}$ and the STAI-S. Participants viewed $23^{2}$ neutral images for two seconds each. Participants viewed one version of each image (e.g., $75 \%$ cropped or $100 \%$ uncropped); half the images were cropped, the other half uncropped. Presentation of cropped and uncropped versions of the images was counterbalanced. The order of image presentation at encoding was randomized. In line with Mathews and Mackintosh (2004), and other boundary restriction studies (Candel et al., 2003; Safer et al., 1998; Takarangi et al., 2015), we did not inform participants that we would test their memory of the images.

Participants viewed one category of images (nature or everyday objects) with noise and one without. Participants rated the pleasantness $(1=$ very unpleasant, $7=$ very pleasant $)$ and emotional arousal ( 1 = not at all emotionally arousing, $7=$ highly emotionally arousing; from Porter, Brinke, Riley, \& Baker, 2014) of each image separately. During a 10-minute delay, participants again completed the STAI-S - measuring their change in arousal after viewing the images - and an unrelated filler task.

\footnotetext{
${ }^{1}$ To examine whether noise condition would moderate the relationship between trait anxiety and boundary errors, we ran hierarchical regressions on proportion of boundary restriction errors and boundary extension errors separately. We found no significant effects, $p s>.05$.

${ }^{2}$ Due to a technical issue, participants only viewed 23 images at encoding.
} 


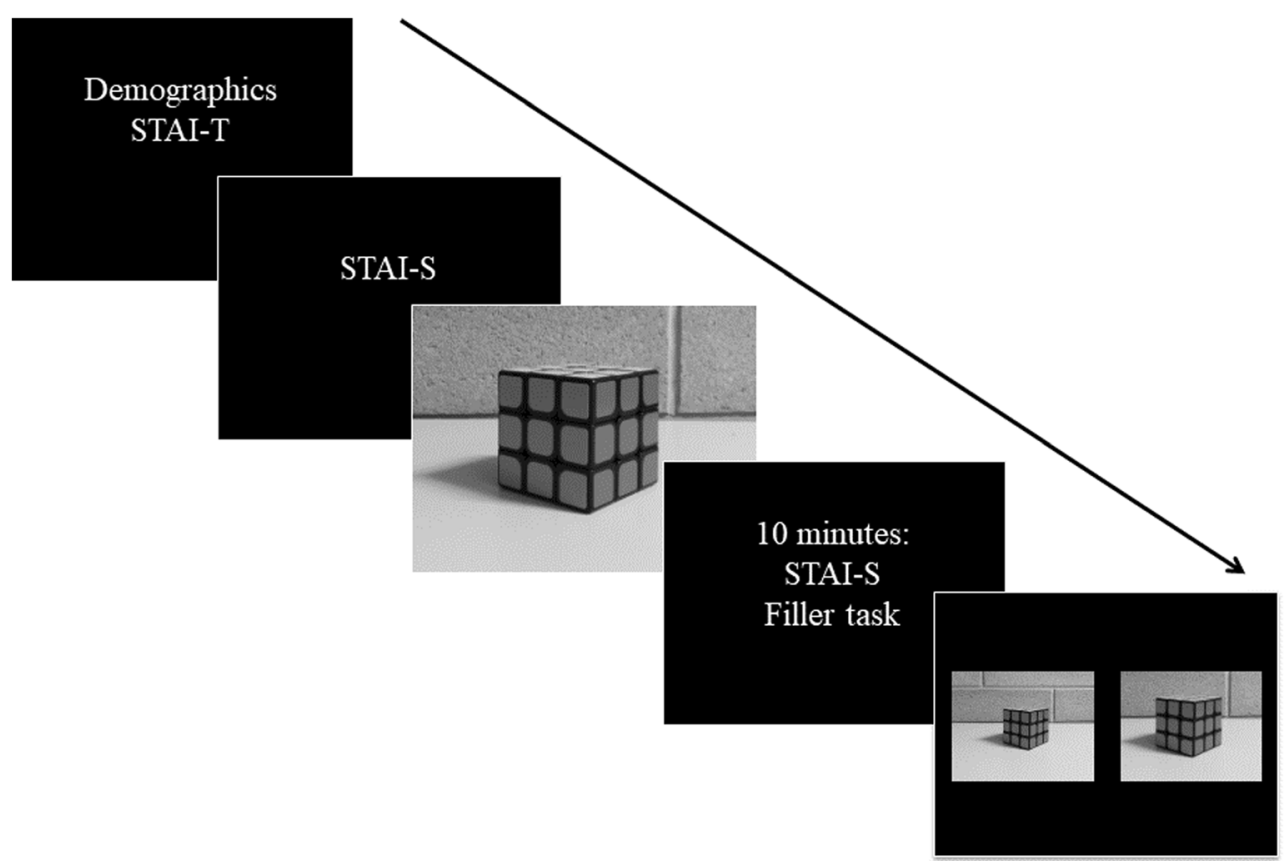

Fig. 1 Diagram of the procedure

Test phase Following the delay, participants saw two versions, side-by-side, of each image they originally viewed. One of these images was in the same format as encoding, the other in the other format (i.e., $75 \%$ cropped vs. $100 \%$ uncropped). This test was similar to previous boundary error studies (Kreindel \& Intraub, 2017; Mathews \& Mackintosh, 2004; Safer et al., 1998). Participants selected which of the two images they originally viewed. Note that for each trial, participants could only either make one type of error (boundary restriction or extension), or be correct. When participants viewed an uncropped image at encoding (half the time), incorrectly selecting the cropped version at test was a boundary restriction error; when participants viewed a cropped image at encoding, incorrectly selecting the uncropped version at test was a boundary extension error. Participants were then debriefed.

\section{Results and discussion}

First, we examined whether noise or image category led participants to rate images as less pleasant and/or more emotionally arousing. Because our design meant that image category and noise exposure were not crossed within-subjects - that is, each participant only saw one category of images with noise and the other category without noise-we could not run a typical $2 \times 2$ within-subjects ANOVA. However, we were able to test whether participants' ratings depended on which image category was paired with noise. We ran a 2 (noise condition: noise, no noise) $\times 2$ (category-noise combination: noise-nature, noise-object) mixed ANOVA on pleasantness ratings. As shown in Fig. 2a, participants rated images as less pleasant when presented with noise, compared to without noise, a main effect for noise condition, $F(1,94)=23.77, p$ $<.001, \eta_{\mathrm{p}}{ }^{2}=.20$. There was no significant interaction between noise condition and category-noise combination, indicating the effect of noise on pleasantness did not depend on which image category the noise was paired with, $F(1,94)=$ $3.82, p=.05, \eta_{\mathrm{p}}{ }^{2}=.04$; and no main effect of category-noise combination $(F<1)$.

We next ran a 2 (noise condition: noise, no noise) $\times 2$ (category-noise combination: noise-nature, noise-object) mixed ANOVA on arousal ratings. As shown in Fig. 2b, participants rated images as similarly arousing with and without noise, $F(1,94)=2.44, p=.12, \eta_{\mathrm{p}}{ }^{2}=.03$. There was also no main effect of category-noise combination $(F<1)$. However, there was a significant interaction between noise condition and category-noise combination, $F(1,94)=18.33, p<.001$, $\eta_{\mathrm{p}}{ }^{2}=.16$. When we examined the effect of noise within category-noise combination using paired-samples $t$ tests, we found that nature images without noise were significantly more arousing than everyday-objects with noise, $t(47)=$ $4.33, p<.001, d=0.63,95 \%$ CI [0.36, 0.99], whereas nature images with noise were not significantly different to everyday objects without noise, $t(47)=1.84, p=.07, d=0.27,95 \% \mathrm{CI}$ $[0.03,0.66]$. This pattern arises because of an underlying difference in arousal ratings where nature images are rated as more arousing than everyday-object images, $t(95)=4.97, p$ $<.001, d=0.51,95 \%$ CI [0.34, 0.78] (see Fig. 2b).

Our data suggest that participants perceived images with noise as more unpleasant but not as more emotionally arousing than images without noise, perhaps because they got used to the noise blasts over time. To test this explanation, we split 

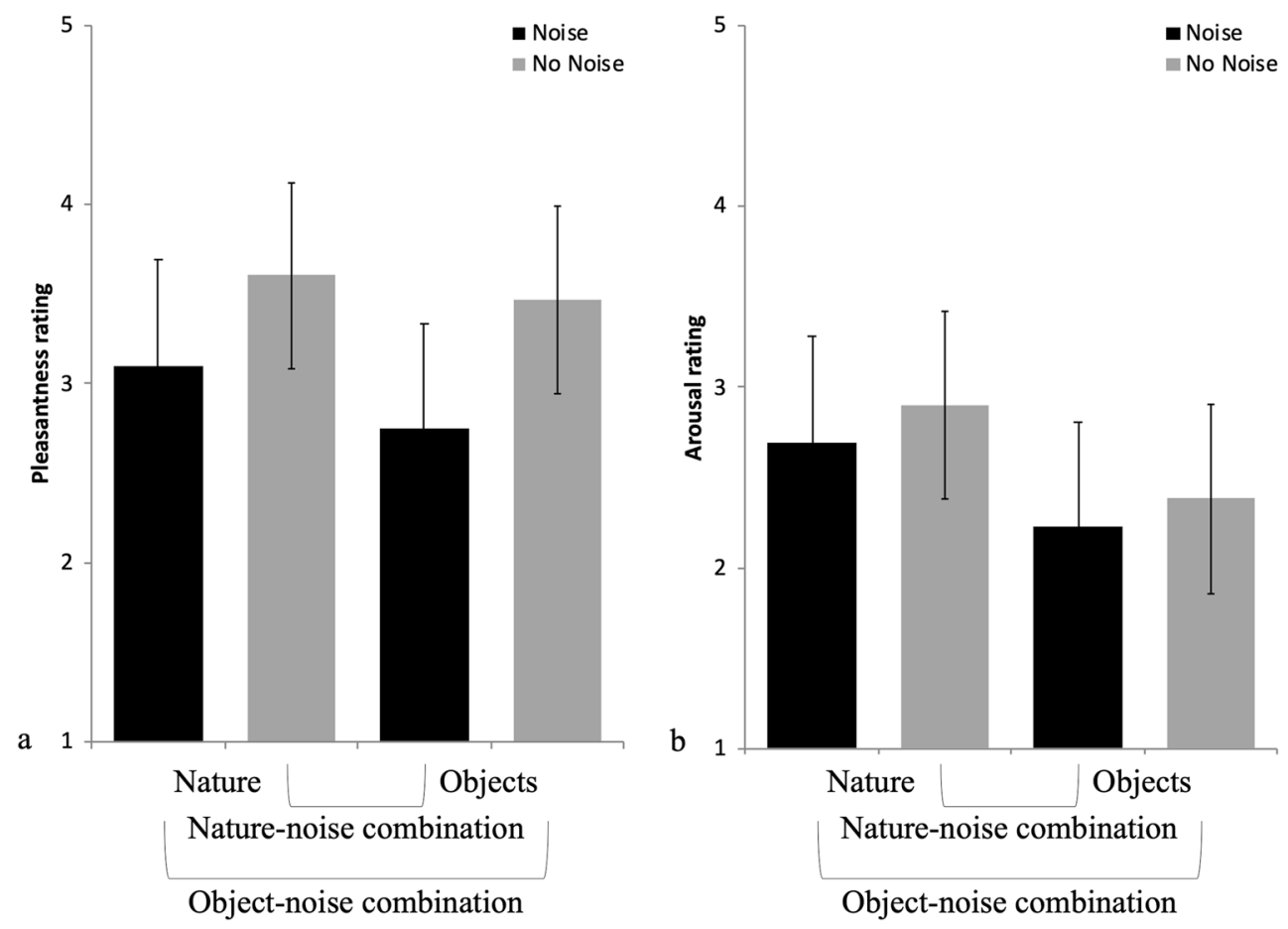

Fig. 2 Mean pleasantness (a) and arousal (b) ratings for category type by noise condition and image-noise combination for Experiment 1a

the data into three time points: the first eight images (Block 1), the second eight images (Block 2), and the third seven images (Block 3) participants viewed. However, arousal ratings did not change significantly over time (Time $1: M=2.43, S D=$ 1.02; Time 2: $M=2.61, S D=1.08$; Time $3: M=2.52, S D=$ $1.05), F(2,190)=2.05, p=.13, \eta_{\mathrm{p}}^{2}=.02$, suggesting that participants were not habituating to the noise blasts.

However, participants' state anxiety (on the STAI-S) increased from Time $1(M=44.63, S D=8.86)$, to Time $2(M$ $=50.81, S D=10.48), t(95)=-8.99, p<.001, d=0.63$, supporting the assertion that overall arousal increased during the encoding phase. We could assume that the change in state arousal was induced by the noise blasts, but it could be due to other factors (e.g., task demands). Without a change in the mean "emotional arousal" ratings for individual photos, we are unable to determine what drove the change in state arousal. However, recall that we did find differences in valence by noise condition, which demonstrates that the noise was changing participants' perception of the valence of images accompanied by noise. The presence of noise may have led to a negatively valenced general state, which persisted across the task and led people to rate the images presented with noise in line with this state (i.e., as unpleasant). Indeed, the effect of white noise on general liking relates to how much the noise itself is liked, rather than the stimulus being rated (e.g., liking of food; Woods et al., 2011). Further, we know that changes in valence persist during a task, while changes in arousal may not (Gomez, Zimmermann, Guttormsen Schär, \& Danuser, 2009). It is possible, then, that any increased arousal induced by the noise did not last across the task. The change in state arousal does not fit with this idea. But this change may have arisen because of other factors. From these data, therefore, we are unable to conclude that the noise changed participants' arousal while viewing the images. Experiment 2 addresses this limitation.

Analysis of boundary errors Examining the proportion of participants' errors overall, we found that participants were inaccurate - essentially at chance - in identifying the correct im$\operatorname{age}^{3}(M=0.46, S D=0.15$, range: $0.13-1)$. Next, we addressed our principal research question: Does arousal increase boundary restriction and attenuate boundary extension? We conducted a 2 (noise: noise, no noise) $\times 2$ (encoded image: cropped, uncropped) $\times 2$ (category-noise combination: noise-nature, noise-object) mixed ANOVA on proportion of errors. There was no difference in the proportion of boundary extension versus boundary restriction errors (i.e., no main effect of encoded image), $F(1,94)=2.64, p=.11, \eta_{\mathrm{p}}^{2}=.03$ (see Fig. 3).

Recall that we predicted that when images were accompanied by noise, participants who saw an uncropped image at encoding would be more likely to make boundary restriction errors and participants who saw a cropped image at encoding would be less likely to make boundary extension errors. Our analysis supported this prediction (see Fig. 3): There was a significant interaction between noise and encoded image, $F(1,94)=33.52, p<.001$, $\eta_{\mathrm{p}}^{2}=.26$. Planned comparisons showed that participants made

\footnotetext{
${ }^{3}$ We asked participants to rate their certainty of their answers and removed responses they reported as guesses. Removing guesses reduced the error rate: $(M=.38, S D=.15)$. But an ANOVA with guesses removed showed the same pattern of results.
} 


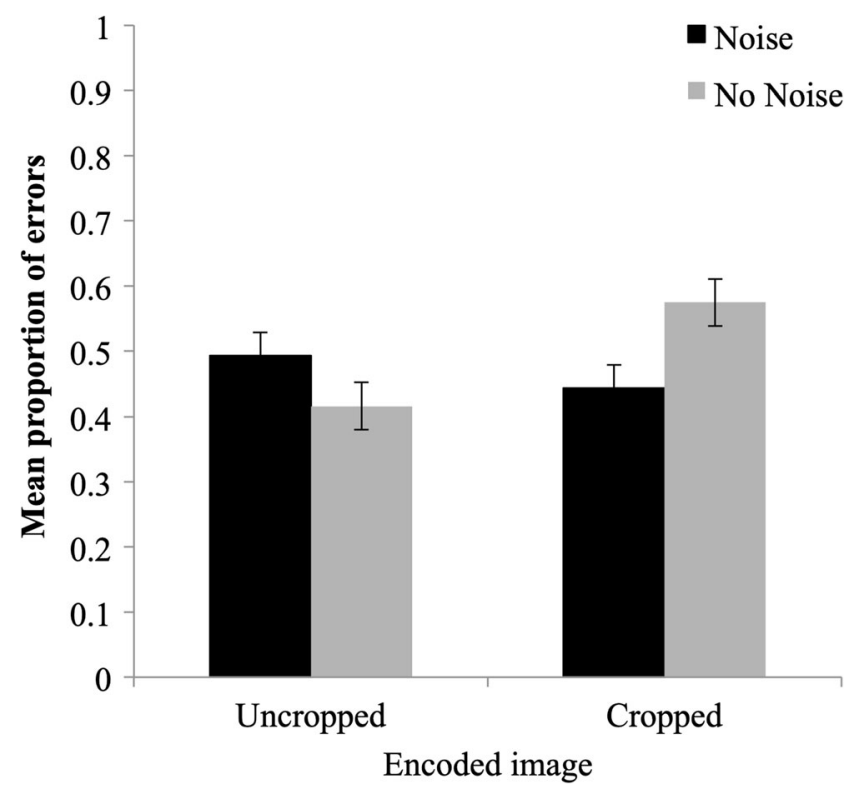

Fig. 3 Mean proportion of boundary errors (with 95\% confidence intervals), by encoded image and noise collapsed across noise-category combination

more boundary restriction errors when cropped images were paired with noise, compared with when they appeared without noise, $t(95)=4.27, p<.001, d=0.26,95 \%$ CI $[0.14,0.39]$. Participants made fewer boundary extension errors when cropped images were paired with noise compared with when they were paired without noise, $t(95)=4.28, p<.001, d=$ $0.53,95 \%$ CI $[0.28,0.80]$. We ran paired-samples Bayesian $t$ tests with default Cauchy prior (Rouder, Speckman, Sun, Morey, $\&$ Iverson, 2009) on these variables and found $\mathrm{BF}_{10}=383.30$ for boundary restriction errors, and $\mathrm{BF}_{10}=377.50$ for boundary extension errors. According to the statistical interpretation that Wetzels et al. (2011) suggest, these Bayes factors indicate decisive evidence that these differences are present. In other words, participants were more likely to incorrectly recognize uncropped images as having narrower boundaries when images were presented with noise, and more likely to recognize cropped images as having more extensive boundaries when images were presented without noise.

We next examined whether these effects depended on category-noise combination. The three-way interaction between category-noise combination and our two primary IVs-encoded image and noise, was not significant, $F(1,94)=2.74, p=.10, \eta_{\mathrm{p}}{ }^{2}=.03$, meaning that the critical interaction between encoded image and noise did not depend on which category the noise was paired with. There were no other significant effects involving category-noise combination $(F s<1)$.

In summary, seeing an image with noise affected how participants remembered the boundaries of that image. One explanation for this pattern is that noise increased arousal, which led to a narrowing of attention to the central details of the image. However, this interpretation depends on how arousal is quantified. Because noise did not increase how participants rated the emotional arousal of the images, we cannot conclude that the underlying mechanism is arousal. We did find that noise increased ratings of image unpleasantness. Thus, it is possible that unpleasantness also leads to a narrowing of attention. Indeed, previous research has found that negative images, compared with neutral images, increase focus on central details versus peripheral details (e.g., Berntsen, 2002; Fawcett et al., 2013; Mathews \& Mackintosh, 2004; Safer et al., 1998; Takarangi et al., 2015). It is also possible that the underlying mechanism is arousal, but our measure did not capture changes in participants' experienced arousal.

Another limitation of this experiment was our use of images from existing databases (IAPS and NAPS). We used these databases because of their available arousal and valence norms. However, there were few images with a central object and expansive background, as required for the present study. Research has found that boundary extension only occurs for images where people could potentially extrapolate beyond the image boundaries (McDunn et al., 2014). Additionally, in selecting the images that best fit our image composition and category match criteria - our nature images $(M=5.40, S D=$ 0.40 , range: $4.70-5.96)$ were more pleasant than everydayobject images $(M=4.90, S D=0.30$, range: $4.43-5.42)$, and more arousing $(M=4.18, S D=0.95$, range: $2.82-5.90)$ than the everyday-object images $(M=2.66, S D=0.52$, range: 1.76-3.44; based on the normed IAPS data on pleasantness and arousal). To address these issues, we sourced new images that gave us the ability to crop the background without any extra details in the periphery, and match across category on valence and arousal. 


\section{Experiment 1b}

\section{Method}

Participants We recruited 102 participants from the Flinders University research participation pool and from the public. Our analyses focused on 96 participants; we excluded participants who did not follow instructions $(n=2)$ or experienced technical problems (e.g., sound inadvertently turned off; $n=$ 4). The majority of participants $(76 \%)$ were female, and Caucasian (including White 69.8\%). The participants ranged in age from 18 to 53 years $(M=23.13, S D=6.89)$. Participants received either course credit, or payment (AUD\$10) for their time.

Design We used the same design as in Experiment 1a.

Materials We used the same anxiety measures ${ }^{4}$ and noise manipulation as in Experiment 1a, but our stimuli were a combination of images from the IAPS, NAPS, and images taken specifically for our study. We selected or edited (using Photoshop) 128 images - in various categories - to have one central object, on an expansive background. We asked 180 pilot participants to rate our new images on valence $(1=$ very pleasant to $5=$ very unpleasant $)$ and arousal $(1=$ relaxed $/$ calm, 5 = aroused/excited $)$. We asked a second sample to categorize the images into distinct categories and rate their category membership. We determined that 48 images fit into the same two distinct categories as we used previously: nature and everyday objects. We selected 12 nature images and 12 everyday-object images based on the best category-fit ratings, and neutral valence and arousal range. Our new image sets were matched on valence (nature: $M=2.81, S D=0.41$, range: 2.00-3.30; objects: $M=2.91, S D=0.11$, range: $2.67-3.04$ ) and arousal (nature: $M=3.23, S D=0.61$, range: $1.62-4.25$; objects: $M=3.24, S D=0.13$, range: $3.07-3.60)$.

Procedure We used the same procedure as in Experiment 1a.

\section{Results and discussion}

We again ran a 2 (noise condition: noise, no noise) $\times 2$ (category-noise combination: noise-nature, noise-object) mixed ANOVA on pleasantness ratings. Like in Experiment 1a, participants rated images as less pleasant when presented with noise, compared with when presented without noise, a main effect for noise condition, $F(1,94)=62.62, p<.001, \eta_{\mathrm{p}}{ }^{2}=.40$ (see Fig. 4a). There was no main effect of category-noise combination $(F<1)$. However, contrary to Experiment 1a,

\footnotetext{
${ }^{4}$ We again examined whether noise condition would moderate the relationship between trait anxiety and boundary errors. We ran hierarchical regressions on the proportion of boundary restriction errors and boundary extension errors separately. We found no significant effects $(p s>.05)$.
}

there was a significant interaction between noise condition and category-noise combination, indicating the that effect of noise on pleasantness did depend on which image category the noise was paired with, $F(1,94)=29.14, p<.001, \eta_{\mathrm{p}}{ }^{2}=.24$. When we examined the effect of noise within category-noise combination, we found that nature images without noise were significantly more pleasant than everyday objects with noise, $t(47)=12.33, p<.001, d=1.78,95 \%$ CI [1.58, 2.19], whereas nature images with noise and everyday-object images without noise were not significantly different, $t(47)=1.49, p=.14, d=$ $0.22,95 \%$ CI $[0.12,0.84]$. As shown in Fig. 2a, this pattern arises because of an underlying difference in pleasantness ratings where nature images were rated in the experiment as more pleasant than everyday-object images, $t(95)=4.20, p$ $<.001, d=0.43,95 \%$ CI $[0.40,1.13]$.

We again ran a 2 (noise condition: noise, no noise) $\times 2$ (category-noise combination: noise-nature, noise-object) mixed ANOVA on arousal ratings. Consistent with Experiment 1a, participants rated images on emotional arousal similarly when presented with noise compared with when presented without noise $(F<1)$, with no main effect of category-noise combination, $F(1,94)=1.60, p<.21, \eta_{\mathrm{p}}{ }^{2}=$ .02 . There was a significant interaction between noise condition and category-noise combination, $F(1,94)=51.38, p<$ $.001, \eta_{\mathrm{p}}{ }^{2}=.35$. Specifically, nature images with noise were rated as higher in arousal than were object images without noise, $t(47)=5.18, p<.001, d=0.75,95 \%$ CI $[0.63,1.43]$, whereas object images with noise were rated as lower in arousal than were nature images without noise, $t(47)=4.96$, $p<.001, d=0.72,95 \%$ CI $[0.61,1.45]$. As shown in Fig. 4 b, this pattern is similar to Experiment 1a: Again, participants rated nature images as more arousing than everyday-object images, $t(95)=7.21, p<.001, d=0.74,95 \%$ CI $[0.75$, 1.31]. This difference was somewhat surprising here, given that we matched this new set of images on arousal ratings.

Again, participants' state anxiety (on the STAI-S) increased from time $1(M=35.95, S D=9.52)$, to time $2(M=41.91, S D=$ $7.78, t(95)=-4.79, p<.001, d=0.49$, supporting the assertion that overall arousal was increased during the encoding phase.

Analysis of boundary errors Examining the proportion of errors overall, we found that participants were often inaccurate in identifying the correct image ${ }^{5}(M=.45, S D=.09$, range: $0.17-0.67)$. We again conducted a 2 (noise: noise, no noise) $\times$ 2 (encoded image: cropped, uncropped) $\times 2$ (category-noise combination: noise-nature, noise-object) mixed ANOVA on proportion of errors. Contrary to Experiment 1a, participants made significantly more boundary extension errors than boundary restriction errors, $F(1,94)=439.55, p<.001, \eta_{\mathrm{p}}{ }^{2}$

\footnotetext{
${ }^{5}$ We asked participants to rate their certainty of their answers and removed responses they reported as guesses. As with Experiment 1a, removing guesses reduced the error rate: $(M=.39, S D=.12)$. But an ANOVA with guesses removed showed the same pattern of results.
} 

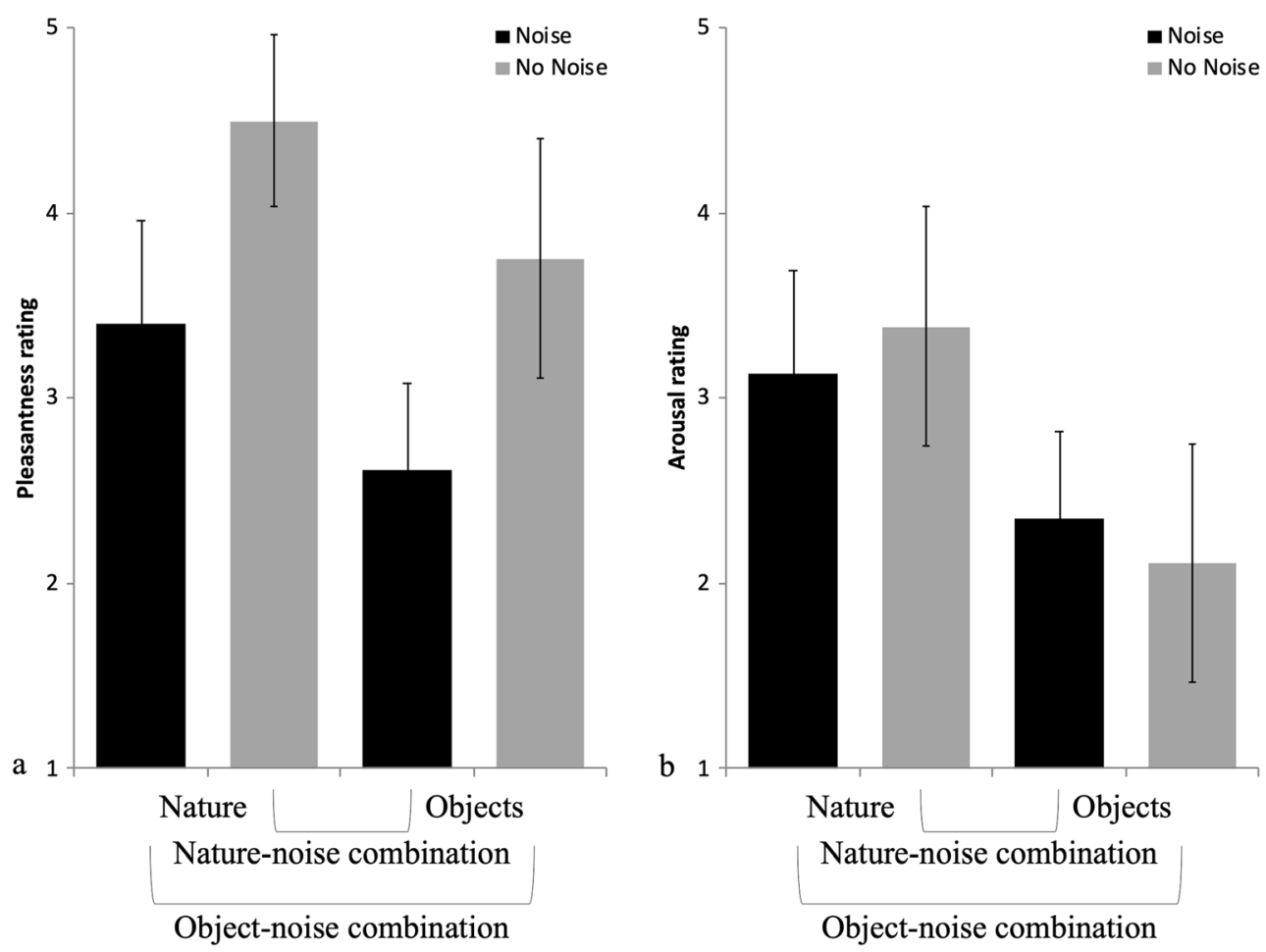

Fig. 4 Mean pleasantness (a) and arousal (b) ratings for category type by noise condition and image-noise combination for Experiment $1 \mathrm{~b}$

$=.82$, which is consistent with previous research on boundary restriction (Candel et al., 2003; Mathews \& Mackintosh, 2004; Safer et al., 1998; Takarangi et al., 2015).

Figure 5 shows boundary errors split by noise and encoded image. We expected that when images were accompanied by noise, participants who saw an uncropped image at encoding would be more likely to make an error at test (boundary restriction) and participants who saw a cropped image at encoding would be less likely to make an error at test (boundary extension). Indeed, like Experiment 1a, there was a significant Noise $\times$ Encoded Image interaction, $F(1,94)=5.10, p=.03 \eta_{\mathrm{p}}^{2}$ $=.051$. As shown in Fig. 5, and consistent with Experiment 1a, planned comparisons showed that participants made significantly more errors for cropped images (boundary restriction errors) when presented with noise compared with when presented without noise, $t(95)=2.39, p=.02, d=0.24, \mathrm{BF}_{10}=1.07$; contrary to Experiment 1a, however, participants made a similar number of errors for uncropped images (boundary extension errors) for

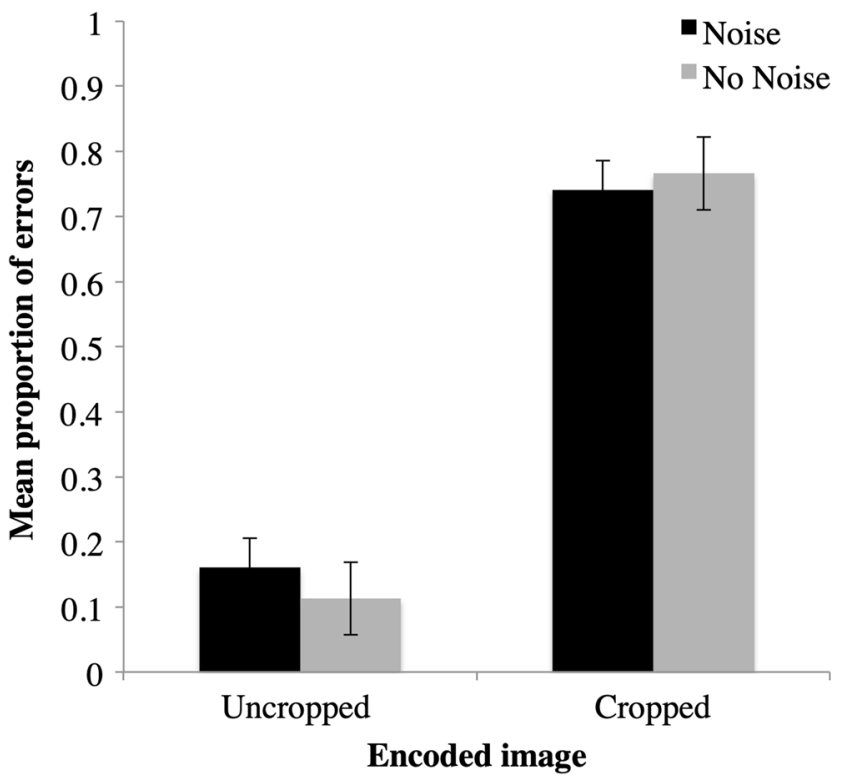

Fig. 5 Mean proportion of boundary errors (with $95 \%$ confidence intervals), by crop type and noise collapsed across category 
images that were presented with and without noise, $t(95)=1.04$, $p=.30, d=0.10, \mathrm{BF}_{10}=.19$.

There was no main effect of category-noise combination $(F<$ $1)$. There was also no three-way interaction between categorynoise combination and encoded image and noise, $F(1,94)=$ 2.96, $p=.09, \eta_{\mathrm{p}}{ }^{2}=.03$. All two-way interactions with category-noise combination were nonsignificant $\left(F_{\mathrm{S}}<1\right)$.

Consistent with Experiment 1a, our data show that images presented with noise resulted in more boundary restriction errors and fewer boundary extension errors compared with those without the noise, but here the difference in boundary extension errors was not significant.

As with Experiment 1a, our arousal question asked participants to rate how emotionally arousing the image was. Thus, participants may not have been introspecting about their own state of arousal, but instead judging the neutral image to be unarousing. Additionally, it should be noted that, like other studies (e.g., Candel et al., 2003; Safer et al., 1998), our participants could only make either a correct response, or a boundary restriction or extension error, depending on whether they viewed the cropped or uncropped image at encoding. Therefore, the type of error they could make was confounded with the view at encoding. This procedure assumed participants' memory matched one of the two views presented at test, which may not necessarily be true. Further, although we found that noise decreased the number of boundary extension errors and noise increased the number of boundary restriction errors, these were only in comparison to "correct" responses. Experiments that investigate boundary extension often use a camera-distance paradigm, ostensibly to avoid confounds such as the one from our two experiments (e.g., Candel et al., 2003; Daniels \& Intraub, 2006; Intraub, 2004; Intraub et al., 2006; Takarangi et al., 2015). With a camera-distance paradigm, participants can make both types of errors for each image. To address these issues, in Experiment 2 we changed the question at encoding to reflect how participants felt while viewing the images, and changed the test to a camera-distance judgement.

\section{Experiment 2}

\section{Method}

Participants We recruited 96 participants from the Flinders University research participation pool and from the public. The majority of participants $(65.6 \%)$ were female, and Caucasian (including White 69.8\%). The participants ranged in age from 18 to 63 years $(M=22.27, S D=6.91)$. Upon completion of the study, participants were reimbursed (AUD\$10) for their time.

Design We used the same design as in Experiments 1a and 1b, with a change in the wording of the arousal measure and a distance judgement at test.
Materials We used the same materials as in Experiment $1 \mathrm{~b} .^{6}$

Procedure We preregistered this study on the Open Science Framework (https://osf.io/cd6wt/). Our procedure was identical to that of Experiment $1 \mathrm{a}$ and $1 \mathrm{~b}$, with two exceptions. During encoding, we asked participants how they felt while viewing the images (arousal: "How did you feel while viewing that image?" 1 = not at all emotionally aroused, 7 = highly emotionally aroused; Betella \& Verschure, 2016), and at test we introduced a cameradistance paradigm. Participants viewed the same images at encoding and at test, and were told that some of the images had been altered (Takarangi et al., 2015). Participants then rated the images as $-2=$ much closer than the original (the participant remembered the original image as having wider boundaries: boundary extension error); $-1=$ slightly closer than the original (boundary extension error); $0=$ no change (no error); 1 = slightly farther than the original (boundary restriction error); or $2=$ much farther than the original (the participant remembered the original image as having more restricted boundaries: boundary restriction error).

\section{Results and discussion}

Again, ${ }^{7}$ we examined whether exposure to noise or a particular category-noise combination led participants to rate images as less pleasant, and feel more emotionally aroused while viewing images with noise. We ran a 2 (noise condition: noise, no noise) $\times 2$ (category-noise combination: noise-nature, noise-object) mixed ANOVA on pleasantness ratings. Like in Experiments $1 \mathrm{a}$ and $1 \mathrm{~b}$, participants rated images as less pleasant when presented with noise, compared with when presented without noise, a main effect for noise condition, $F(1,94)=48.52$, $p<.001, \eta_{\mathrm{p}}{ }^{2}=.34$ (see Fig. 6a). Consistent with Experiment $1 \mathrm{a}$ and contrary to Experiment $1 \mathrm{~b}$, there was no significant interaction between noise condition and category-noise combination, indicating that the effect of noise on pleasantness did not depend on which image category the noise was paired with. There was no main effect of category-noise combination $(F<1)$.

We next ran a 2 (noise condition: noise, no noise) $\times 2$ (category-noise combination: noise-nature, noise-object) mixed ANOVA on arousal ratings. Contrary to Experiment $1 \mathrm{a}$ and $1 \mathrm{~b}$, participants reported feeling higher emotional arousal when viewing the images with noise, compared with when viewing without noise, $F(1,94)=136.76, p<.001, \eta_{\mathrm{p}}{ }^{2}$ $=.59$ (see Fig. 6b). There was also no significant interaction between noise condition and category-noise combination,

\footnotetext{
${ }^{6}$ We again examined whether noise condition would moderate the relationship between trait anxiety and boundary errors. We ran hierarchical regressions on the proportion of boundary restriction errors and boundary extension errors separately. We found no significant effects $(p s>.05)$.

${ }^{7}$ Note that we did not pre-register analyses that were not testing our hypothesis directly, but these analyses are in line with Experiments 1a and 1b.
} 

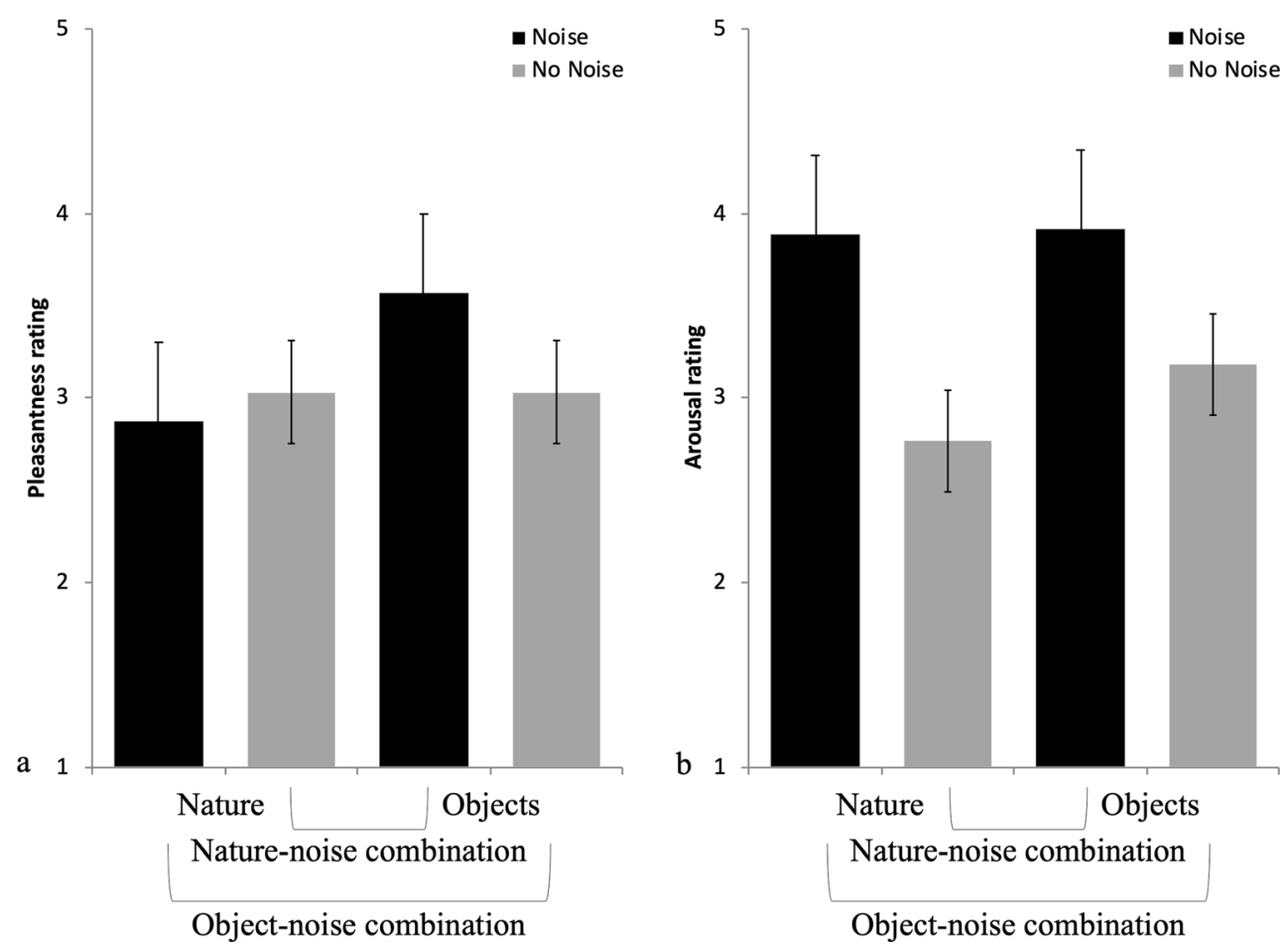

Fig. 6 Mean pleasantness and arousal ratings for category type by image-noise combination for Experiment 2

indicating that the effect of noise on reported arousal did not depend on category-noise combination, and no main effect of category-noise combination $(F \mathrm{~s}<1)$.

Importantly then, when participants rated their emotional arousal after each image - rather than arousal specific to the images themselves - we found what we had originally predicted: that participants experienced more emotional arousal when viewing images with noise than when viewing images without noise.

Additionally, consistent with Experiments 1a and 1b, participants' state anxiety (on the STAI-S) increased from Time 1 $(M=37.29, S D=9.32)$, to Time $2(M=42.26, S D=11.02)$, $t(95)=-5.90, p<.001, d=0.49$, showing that overall arousal was again increased during the encoding phase.

Analysis of boundary errors We first examined how often-on average - participants judged the images as much closer $(3.95 \%)$, slightly closer $(29.30 \%)$, no change $(58.94 \%)$, slightly farther $(5.95 \%)$, and much farther (1.87\%). Participants were correct more than half the time in recognizing no change to the images. ${ }^{8}$ We then calculated the mean reported camera distance for each participant on the -2 to +2 scale across all images $(M=-0.09 S D=0.18)$, and conducted an exploratory one-samples $t$ test on this variable (Beighley et al., 2018; Intraub et al., 1996; Takarangi et al., 2015). We found that the mean camera distance was significantly less than zero (where zero indicates no error), $t(95)=-5.00, p<.001, d=$

\footnotetext{
${ }^{8}$ We asked participants to rate their certainty of their answers and removed responses they reported as guesses. As with the earlier experiments, removing guesses reduced the error rate $(M=.35, S D=.21)$. But an ANOVA with guesses removed showed the same pattern of results.
}

$0.51,95 \% \mathrm{CI}[0.06,0.13]$. Therefore, when participants made errors, those errors tended to be boundary extension.

Next, we classified average camera distance judgements by noise condition and category to address our principal research question: Does arousal increase boundary restriction and attenuate boundary extension? Figure 7 displays these data. According to our preregistered analysis plan, we ran a 2 (noise: noise, no noise) $\times 2$ (category-noise combination: noise-nature images, noise-everyday-object images) mixed ANOVA on mean camera-distance judgments, which revealed that when images appeared with noise, they were less likely to be judged as closer than the original compared with when images appeared without noise, a significant main effect of noise, $F(1,94)=13.58$, $p<.001, \eta_{\mathrm{p}}^{2}=.13, \mathrm{BF}_{10}=0.72$. There was no main effect of category-noise combination, nor a significant interaction between noise and category-noise combination $\left(F_{\mathrm{S}}<1\right)$.

Overall, these data suggest that noise attenuated the extent of boundary extension. To examine the absolute number of errors that participants made, and - in line with Takarangi et al. (2015) and Beighley et al. (2018) - whether noise affected the rare occasions where participants made boundary restriction errors, we determined the error rate for boundary restriction and boundary extension. We calculated proportions by dividing the number of test images on which participants made a particular type of error (i.e., $\mathrm{BE}=$ distance judgements $<0 ; \mathrm{BR}=$ distance judgements $>$ 0 ) by the total number of test images. We then ran the preregistered 2 (noise: noise, no noise) $\times 2$ (noise-category combination: noise with nature, noise with objects) $\times 2$ (error type: BE, BR) mixed ANOVA. This analysis showed that participants were more likely to make boundary extension errors (incorrectly 


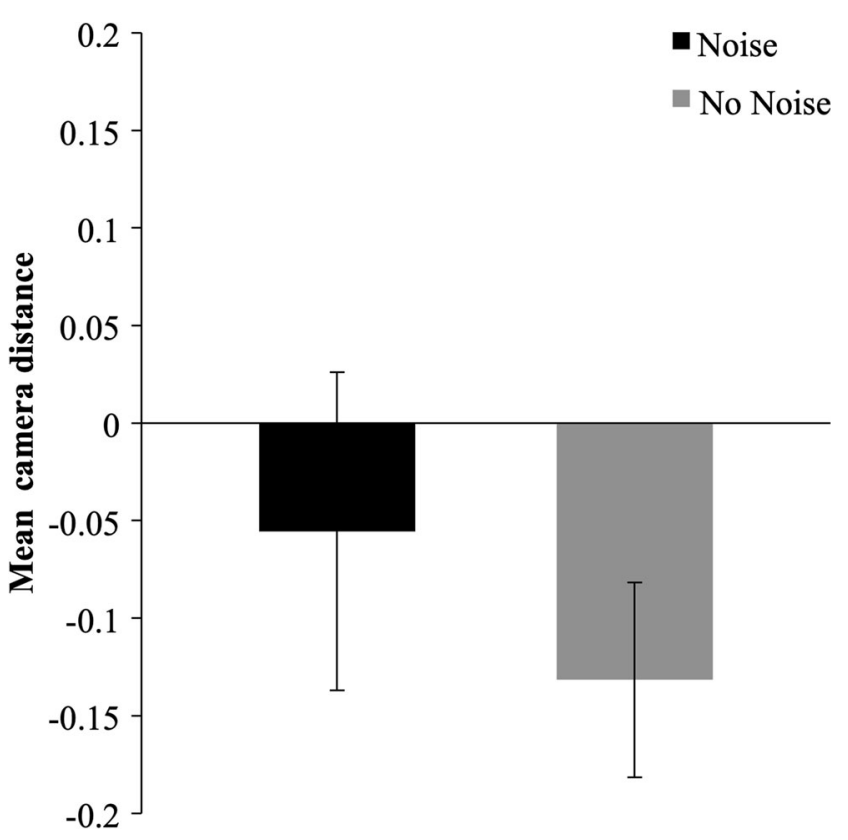

Fig. 7 Mean camera distance by noise condition

judging the images as closer at test; $17 \%$ of trials) than boundary restriction errors (incorrectly judging the images as farther at test; $4 \%$ of trials). This effect of error type was the only significant effect in the model, $F(1,94)=72.24, p<.001, \eta_{\mathrm{p}}{ }^{2}=.44$; there was no main effect of noise condition, $F(1,94)=1.43, p=.24$, $\eta_{\mathrm{p}}{ }^{2}=.02$; and no significant interaction between noise and category-noise combination, $F(1,94)=1.64, p=.20, \eta_{\mathrm{p}}{ }^{2}=$ $.02(F \mathrm{~s}<1$ for all other effects). Thus, the presence of noise did not affect the frequency of boundary extension or boundary restriction errors when operationalized as the proportion of total test items. Indeed, boundary restriction errors in particular were very low. This result is inconsistent with the pattern of data revealed by the mean distance judgements. However, we propose that the mean distance judgement is a more sensitive measure, because it takes into account the rate at which participants make both types of errors, and also the extent of those errors (e.g., "slightly closer" vs. "much closer").

In short, the data from Experiment 2 suggest that noise attenuates boundary extension, based on participants' average distance judgments. However, noise does not affect the absolute number of distance errors - whether restriction or extensionthat participants make. Based on participants reporting higher arousal in response to images presented with noise, we interpret these data as noise eliciting high state arousal.

\section{General discussion}

To summarize, over three experiments we found that images presented with noise were less pleasant compared with images presented without noise. Further, exposure to noise increased state anxiety in all three experiments; in Experiment
2, participants' emotional arousal was higher when viewing images with noise compared with when viewing images without noise. In Experiment 1a, images presented with noise led to more boundary restriction errors and fewer boundary extension errors (compared with images without noise). In Experiment 1b, noise led to more boundary restriction errors, and in Experiment 2, we found that noise attenuated boundary extension. Taken together, our data suggest that noise can lead to both a reduction in boundary extension and an increase in boundary restriction. Our findings are consistent with the idea that heightened arousal, which may be accompanied by lowered pleasantness, increases a person's perception of threat (Cole, Balcetis, \& Dunning, 2013). Increased focus on threat may result in selective attention to central objects in a scene, in order to locate potential danger. Indeed, previous research shows that threat is associated with an immediate fear response, which focuses attention toward threatening objects (Koster, Crombez, Van Damme, Verschuere, \& De Houwer, 2004; Schmidt, Belopolsky, \& Theeuwes, 2015; Van Damme, Crombez, \& Notebaert, 2008), and produces a feeling of closer proximity to the object (Cole et al., 2013). Here, we found - consistent with Levine and Edelstein (2009), Mathews and Mackintosh (2004), and Safer et al. (1998) - that arousal may also lead to an increased tendency to remember an image with narrower boundaries. An increased focus on threat (or perceived threat) may also reduce people's opportunity-and available resources - to extrapolate beyond the boundaries of an image. This process, in turn, reduces the likelihood of internally generating additional mental representations, and thus of making a source-monitoring error that results in boundary extension. 
Importantly, although we aimed to isolate arousal from the other qualities of a scene, our noise manipulation also affected how pleasant participants found the neutral images, in addition to affecting the images' emotional arousal. Thus, the proposed mechanism of increased focus toward central, threatening aspects of a scene could arise from negative valence, increased arousal, or a combination of the two.

Our findings have methodological implications. Previous studies may have failed to find boundary restriction because of how arousal was manipulated (e.g., using negative images; see Mathews \& Mackintosh, 2004). We instead used an external manipulation of arousal along with neutral images rather than relying on negative images to elicit arousal. This procedure meant other aspects of the image were consistent (e.g., content, context, composition), and is thus a more methodologically sound way of manipulating the arousal of an image (Porter et al., 2014; also see Beighley et al., 2018). Indeed, noise consistently elicits an arousal response similar to fear across individuals (Rhudy \& Meagher, 2001). By contrast, how unpleasant a negative image is perceived to be may vary considerably across individuals - for example, according to their age (Charles, Reynolds, \& Gatz, 2001), gender (Collignon et al., 2011; Davis et al., 2012; Felmingham, Tran, Fong, \& Bryant, 2012; McRae, Ochsner, Mauss, Gabrieli, \& Gross, 2008), cultural background (Davis et al., 2012), anxiety diagnosis (Cook, Melamed, Cuthbert, McNeil, \& Lang, 1988), or personality (Lang, Greenwald, Bradley, \& Hamm, 1993). Further, because eyewitness testimony, for example, is dependent on visual memory accuracy and is often tied to highly arousing events, we can see potential applications for our findings in that context.

Our study has limitations. First, although we did not control the number of times the noise was presented in a row, we randomized the presentation for every participant to minimize habituation to the noise. Second, the majority of our participants were female. Previous research has shown that arousal can improve the recollection of negative (vs. neutral) images among females versus males (Felmingham et al., 2012). Thus, the difference in reaction to emotional arousal could lead to different patterns of boundary judgement errors. However, we were reluctant to investigate sex differences with our data due to the low number of males. Further research could address this issue. Third, it may be useful to measure induced arousal objectively, such as with a Galvanic Skin Response measure (Rhudy \& Meagher, 2001), to confirm a temporal rise in emotional arousal without relying on self-report. Fourth, our manipulation only investigated the effect of negative arousal on boundary judgement errors. Future research should investigate whether these effects can be distinguished from positive arousal.

Finally, we intentionally confounded noise and image category within version to produce a conditioning response (see Dunsmoor et al., 2015), and in some cases, there were differences between the noise-category combinations. Further, we found that participants rated the nature images as more pleasant in Experiment $1 \mathrm{~b}$ and more arousing in Experiments 1a and $1 \mathrm{~b}$. Indeed, the potential composition differences between the image categories could have implications for boundary judgements. For example, some researchers have posited that boundary extension errors are the result of participants imagining and extrapolating beyond the periphery (Intraub \& Dickinson, 2008; see Hubbard, Hutchison, \& Courtney, 2010, for a review). Participants may be more likely to contextualize something that exists in an unseen wider world (on a table, in a room - as in our object images) — which would potentially lead to more boundary extension. Participants may be less likely to contextualize something that has more "wide world" information (such as a house, in a field, as in our nature images) — which would potentially lead to less boundary extension. However, in a recent experiment, Munger and Multhaup (2016) did not find any differences in image type (i.e., various outdoor scenes and various object images) and the number and extent of boundary errors that were made. Yet Munger and Multhaup found that imagining isolated objects improves memory, reducing source memory errors. Therefore, it is possible that participants treat object images (where an object is available to be visually represented), and nature images (where there is no specific "object" to have a visual representation of) differently. To examine the effect of arousal arising specifically from composition differences between image categories, future research could compare nature images with object images in the absence of an arousing stimulus. Furthermore, to investigate whether conditioning arousal to one category of images is necessary, future research could instead use only one category of neutral stimuli.

To summarize, our results reveal that images presented with noise led to more boundary restriction errors and fewer boundary extension errors than images presented without noise. Although our data fit well within the literature on boundary judgements, our results also indicate that arousal may be an important mechanism in the boundary restriction phenomenon —one that has not been examined in isolation until now.

\section{References}

Anderson, S. F., Kelley, K., \& Maxwell, S. E. (2017). Sample-size planning for more accurate statistical power: A method adjusting sample effect sizes for publication bias and uncertainty. Psychological Science, 28(11), 1547-1562. https://doi.org/10.1177/ 0956797617723724

Beighley, S., Sacco, G. R., Bauer, L., Hayes, A. M., \& Intraub, H. (2018). Remembering: Does the emotional content of a photograph affect boundary extension?. Emotion. Advanced online publication. https://doi.org/10.1037/emo0000477

Berntsen, D. (2002). Tunnel memories for autobiographical events: Central details are remembered more frequently from shocking than 
from happy experiences. Memory \& Cognition, 30(7), 1010-1020. https://doi.org/10.3758/BF03194319

Betella, A., \& Verschure, P. F. (2016). The affective slider: A digital selfassessment scale for the measurement of human emotions. PLoS ONE, 11(2), e0148037. https://doi.org/10.1371/journal.pone. 0148037

Blackmore, S. J., Brelstaff, G., Nelson, K., \& Troscianko, T. (1995). Is the richness of our visual world an illusion? Transsaccadic memory for complex scenes. Perception, 24, 10751081. https://doi.org/10.1068/ p241075

Bowers, D., \& Heilman, K. M. (1980). Pseudoneglect: Effects of hemispace on a tactile line bisection task. Neuropsychologia, 18(4/5), 491-498. https://doi.org/10.1016/0028-3932(80)90151-7

Callaway, E., \& Dembo, D. (1958). Narrowed attention: A psychological phenomenon that accompanies a certain physiological change. Archives of Neurology and Psychiatry, 79(1), 74-90. https://doi. org/10.1001/archneurpsyc. 1958.02340010092008

Candel, I., Merckelbach, H., \& Zandbergen, M. (2003). Boundary distortions for neutral and emotional pictures. Psychonomic Bulletin \& Review, 10(3), 691-695. https://doi.org/10.3758/BF03196533

Chapman, P., Ropar, D., Mitchell, P., \& Ackroyd, K. (2005). Understanding boundary extension: Normalization and extension errors in picture memory among adults and boys with and without Asperger's syndrome. Visual Cognition, 12(7), 1265-1290. https:// doi.org/10.1080/13506280444000508a

Charles, S. T., Reynolds, C., \& Gatz, M. (2001). Age-related differences and change in positive and negative affect over 23 years. Journal of Personality and Social Psychology, 80, 136-151. https://doi.org/10. 1037/0096-3445.132.2.310

Christianson, S. A., \& Loftus, E. F. (1987). Memory for traumatic events. Applied Cognitive Psychology, 1(4), 225-239. https://doi.org/10. 1002/acp.2350010402

Christianson, S. A., \& Loftus, E. F. (1991). Remembering emotional events: The fate of detailed information. Cognition and Emotion, 5(2), 81-108. https://doi.org/10.1080/02699939108411027

Christianson, S. A., Loftus, E. F., Hoffman, H., \& Loftus, G. R. (1991). Eye fixations and memory for emotional events. Journal of Experimental Psychology: Learning, Memory, and Cognition, 17, 693-701. https://doi.org/10.1037/0278-7393.17.4.693

Cole, S., Balcetis, E., \& Dunning, D. (2013). Affective signals of threat increase perceived proximity. Psychological Science, 24(1), 34-40. https://doi.org/10.1177/0956797612446953

Collignon, O., Girard, S., Gosselin, F., Saint-Amour, D., Lepore, F., \& Lassonde, M. (2011) Women process multisensory emotion expressions more efficiently than men. Neuropsychologia, 48(1), 220-225. https://doi.org/10.1016/j.neuropsychologia.2009.09.007

Cook, E. W., Melamed, B. G., Cuthbert, B. N., McNeil, D. W., \& Lang, P. J. (1988). Emotional imagery and the differential diagnosis of anxiety. Journal of Consulting and Clinical Psychology, 56(5), 734 740. https://doi.org/10.1037/0022-006X.56.5.734

Daniels, K. K., \& Intraub, H. (2006). The shape of view: Are rectilinear views necessary to elicit boundary extension? Visual Cognition, 14(2), 129-149. https://doi.org/10.1080/13506280500460563

Davis, E., Greenberger, E., Charles, S., Chen, C., Zhao, L., \& Dong, Q. (2012). Emotion experience and regulation in China and the United States: How do culture and gender shape emotion responding? International Journal of Psychology, 47 (3), 230-239. https://doi. org/10.1080/00207594.2011.626043

Dickinson, C., \& Intraub, H. (2009). Spatial asymmetries in viewing and remembering scenes: Consequences of an attentional bias? Attention, Perception, \& Psychophysics, 71(6), 1251-262. https:// doi.org/10.3758/APP.71.6.1251

Dunsmoor, J. E., Murty, V. P., Davachi, L., \& Phelps, E. A. (2015). Emotional learning selectively and retroactively strengthens memories for related events. Nature, 520, 345-348. https://doi.org/10. 1038/nature14106
Echterhoff, G., \& Wolf, O. T. (2012). The stressed eyewitness: The interaction of thematic arousal and post-event stress in memory for central and peripheral event information. Frontiers in Integrative Neuroscience, 6, 57. https://doi.org/10.3389/fnint.2012.00057

Fawcett, J. M., Russell, E. J., Peace, K. A., \& Christie, J. (2013). Of guns and geese: A meta-analytic review of the 'weapon focus' literature. Psychology, Crime \& Law, 19, 35-66. https://doi.org/10.1080/ 1068316X.2011.599325

Felmingham, K. L., Tran, T. P., Fong, W. C., \& Bryant, R. A. (2012). Sex differences in emotional memory consolidation: The effect of stressinduced salivary alpha amylase and cortisol. Biological Psychology, 89, 539-544. https://doi.org/10.1016/j.biopsycho.2011.12.006

Gilbert, P. (2001). Evolutionary approaches to psychopathology: The role of natural defences. Australian and New Zealand Journal of Psychiatry, 35(1), 17-27. https://doi.org/10.1046/j.1440-1614. 2001.00856.x

Gomez, P., Zimmermann, P. G., Guttormsen Schär, S., \& Danuser, B. (2009). Valence lasts longer than arousal: Persistence of induced moods as assessed by psychophysiological measures. Journal of Psychophysiology, 23(1), 7-17. https://doi.org/10.1027/0269-8803. 23.1.7

Gottesman, C., \& Intraub, H. (1999). Wide-angle memories of close-up scenes: A demonstrations of boundary extension. Behaviour Research Methods, Instruments, \& Computers, 31(1), 86-93. https://doi.org/10.3758/BF03207697

Grös, D. F., Antony, M. M., Simms, L. J., \& McCabe, R. E. (2007). Psychometric properties of the State-Trait Inventory for Cognitive and Somatic Anxiety (STICSA): Comparison to the State-Trait Anxiety Inventory (STAI). Psychological Assessment, 19(4), 369381. https://doi.org/10.1037/1040-3590.19.4.369

Hubbard, T. L., Hutchison, J. L., \& Courtney, J. R. (2010). Boundary extension: Findings and theories. The Quarterly Journal of Experimental Psychology, 63(8), 1467-1494. https://doi.org/10. 1080/17470210903511236

Intraub, H. (2004). Anticipatory spatial representation of 3D regions explored by sighted observers and a deaf-and-blind-observer. Cognition, 94(1), 19-37. https://doi.org/10.1016/j.cognition.2003. 10.013

Intraub, H. (2010). Rethinking scene perception: A multisource model. Psychology of Learning and Motivation, 52(6), 231-264. https:// doi.org/10.1016/S0079-7421(10)52006-1

Intraub, H. (2012). Rethinking visual scene perception. Wiley Interdisciplinary Reviews: Cognitive Science, 3(1), 117-127. https://doi.org/10.1002/wcs.149

Intraub, H., \& Bodamer, J. L. (1993). Boundary extension: Fundamental aspect of pictorial representation or encoding artifact? Journal of Experimental Psychology: Learning, Memory, and Cognition, 19(6), 1387-1397. https://doi.org/10.1037/0278-7393.19.6.1387

Intraub, H., Daniels, K. K., Horowitz, T. S., \& Wolfe, J. M. (2008). Looking at scenes while searching for numbers: Dividing attention multiplies space. Perception \& Psychophysics, 70(7), 1337-1349. https://doi.org/10.3758/PP.70.7.1337

Intraub, H., \& Dickinson, C. A. (2008). False memory $1 / 20^{\text {th }}$ second later. Psychological Science, 19(10), 1007-1014. https://doi.org/10.1111/ j.1467-9280.2008.02192.x

Intraub, H., Gottesman, C. V., Willey, E. V., \& Zuk, I. J. (1996). Boundary extension for briefly glimpsed images: Do common perceptual processes result in unexpected memory distortions? Journal of Memory and Language, 35, 118-134. https://doi.org/10.1006/jmla.1996. 0007

Intraub, H., Hoffman J., Wetherhold C., \& Stoehs, S.-A. (2006). More than meets the eye: The effect of planned fixations on scene representation. Perception \& Psychophysics, 68(5), 759-769. https://doi. org/10.3758/BF03193699 
Koster, E. H., Crombez, G., Van Damme, S., Verschuere, B., \& De Houwer, J. (2004). Does imminent threat capture and hold attention? Emotion, 4(3), 312. https://doi.org/10.1037/1528-3542.4.3.312

Kramer, T. H., Buckhout, R., \& Eugenio, P. (1990). Weapons focus, arousal, and eyewitness memory: Attention must be paid. Law and Human Behaviour, 14(2), 167-184. https://doi.org/10.1007/ BF01062971

Kreindel, E., \& Intraub, H. (2017). Anticipatory scene representation in preschool children's recall and recognition memory. Developmental Science, 20(5), e12444. https://doi.org/10.1111/desc.12444

Lang, P. J. (2010). Emotion and motivation: Toward consensus definitions and a common research purpose. Emotion Review, 2(3), 229233. https://doi.org/10.1177/1754073910361984

Lang, P. J., Bradley, M. M., \& Cuthbert, B. N. (2008). International Affective Picture System (IAPS): Affective ratings of pictures and instruction manual (Technical Report No. A-8). Gainesville: University of Florida Gainesville.

Lang, P. J., Greenwald, M. K., Bradley, M. M., \& Hamm, A. O. (1993). Looking at pictures: Affective, facial, visceral, and behavioral reactions. Psychophysiology, 30, 261-273. https://doi.org/10.1111/j. 1469-8986.1993.tb03352.x

Levine, L. J., \& Edelstein, R. S, (2009). Emotion and memory narrowing: A review and goal-relevance approach. Cognition \& Emotion, 23(5), 833-875. https://doi.org/10.1080/02699930902738863

Marchewka, A., Zurawski, L., Jednorog, K., \& Grabowska, A. (2014). The Nencki Affective Picture System (NAPS): Introduction to a novel, standardised, wide-range, high-quality, realistic database. Behaviour Research Methods, 46(2), 596-610. https://doi.org/10. 3758/s13428-013-0379-1

Mather, M. (2007). Emotional arousal and memory binding: An objectbased framework. Perspectives on Psychological Science 2(1), 33 52. https://doi.org/10.1111/j.1745-6916.2007.00028.x

Mathews, A., \& Mackintosh, B. (2004). Take a closer look: Emotion modifies the boundary extension effect. Journal of Emotion, 4(1), 36-45. https://doi.org/10.1037/1528-3542.4.1.36

Mathews, A., \& MacLeod, C. (1994). Cognitive approaches to emotion and emotional disorders. Annual Review of Psychology, 45, 25-50. https://doi.org/10.1146/annurev.psych.45.1.25

McDunn, B. A., Siddiqui, A. P., \& Brown, J. M. (2014). Seeking the boundary of boundary extension. Psychonomic Bulletin and Review, 21, 370-375. https://doi.org/10.3758/s13423-013-0494-0

McRae, K., Ochsner, K. N., Mauss, I. B., Gabrieli, J. J., \& Gross J. J. (2008). Gender differences in emotion regulation: An fMRI study of cognitive reappraisal. Group Processes \& Intergroup Relations, 11(2), 143-162. https://doi.org/10.1177/1368430207088035

Ménétrier, E., Didierjean, A., \& Vieillard, S. (2013). Is boundary extension emotionally selective?. The Quarterly Journal of Experimental Psychology, 66(4), 635-641. https://doi.org/10.1080/17470218. 2013.764332

Munger, M. P., \& Multhaup, K. S. (2016). No imagination effect on boundary extension. Memory \& Cognition, 44(1), 73-88. https:// doi.org/10.1177/2041669517724808

Porter, S., Brinke, L. T., Riley, S. N., \& Baker, A. (2014). Prime time news: The influence of primed positive and negative emotion on susceptibility to false memories. Cognition \& Emotion, 28(8), 1422-1434. https://doi.org/10.1080/02699931.2014.887000

Rhudy, J. L., \& Meagher, M. W. (2001). The role of emotion in pain modulation. Current Opinion in Psychiatry, 14(3), 241-245. https://doi.org/10.1097/00001504-200105000-00012

Rosch, E. (1973). Natural categories. Cognitive Psychology, 4(3), 328 350. https://doi.org/10.1016/0010-0285(73)90017-0

Rouder, J. N., Speckman, P. L., Sun, D., Morey, R. D., \& Iverson, G. (2009). Bayesian $t$-tests for accepting and rejecting the null hypothesis. Psychonomic Bulletin \& Review, 16, 225-237.

Safer, M. A., Christianson, S. A., Autry, M. W., \& Osterlund. (1998). Tunnel memory for traumatic events. Journal of Applied Cognitive Psychology, 12, 99-117. https://doi.org/10.1002/(SICI)10990720(199804)12:2<99::AID-ACP509>3.0.CO;2-7

Schmidt, L. J., Belopolsky, A. V., \& Theeuwes, J. (2015). Attentional capture by signals of threat. Cognition and Emotion, 29(4), 687694. https://doi.org/10.1080/02699931.2014.924484

Seamon, J. G., Luo, C. R., Kopecky, J. J., Price, C. A., Rothschild, L., Fung, N. S., \& Schwartz, M. A. (2002). Are false memories more difficult to forget than accurate memories? The effect of retention interval on recall and recognition. Memory \& Cognition, 30(7), 1054-1064. https://doi.org/10.3758/BF03194323

Simons, D. J., \& Levin, D. T. (1998). Failure to detect changes to people during a real-world interaction. Psychonomic Bulletin \& Review, 5(4), 644-649. https://doi.org/10.3758/BF03208840

Spielberger, C. D., Gorsuch, R. L., \& Lushene, R. E. (1970). Manual for the State-Trait Anxiety Inventory. Palo Alto, CA: Consulting Psychologists Press.

Takarangi, M. K. T., Oulton, J. M., Green, D. M., \& Strange, D. (2015). Boundary restriction for negative emotional images is an example of memory amplification. Clinical Psychological Science, 4(1), 82-95. https://doi.org/10.1177/2167702615569912

Van Damme, S., Crombez, G., \& Notebaert, L. (2008). Attentional bias to threat: A perceptual accuracy approach. Emotion, 8(6), 820. https:// doi.org/10.1037/a0014149

Vuilleumier, P. (2005). How brains beware: Neural mechanisms of emotional attention. Trends in Cognitive Sciences, 9(12), 585-594. https://doi.org/10.1016/j.tics.2005.10.011

Waring, J. D., Payne, J. D., Schacter, D L., \& Kensinger, E. A. (2010). Impact of individual differences upon emotion-induced memory trade-offs. Cognition and Emotion, 24(1), 150-167. https://doi.org/ 10.1080/02699930802618918

Wetzels, R., Matzke, D., Lee, M. D., Rouder, J. N., Iverson, G. J., \& Wagenmakers, E. J. (2011). Statistical evidence in experimental psychology: An empirical comparison using $855 t$ tests. Perspectives on Psychological Science, 6, 291-298. https://doi. org/10.1177/1745691611406923.

Woods, A. T, Poliakoff, E., Lloyd, D. M., Kuenzel, J., Hodson, R., Gonda, H., .. . Thomas, A. (2011). Effect of background noise on food perception. Food Quality and Preference, 22(1), 42-47. https:// doi.org/10.1016/j.foodqual.2010.07.003

Publisher's note Springer Nature remains neutral with regard to jurisdictional claims in published maps and institutional affiliations. 